A microgenetic analysis of the relationship between speech and gesture in children: Evidence for semantic and temporal asynchrony

Running head: Relationship between speech and gesture

Dr. Karen J. Pine*^,

Nicola Lufkin* Elizabeth Kirk *\& David Messer**

* School of Psychology, University of Hertfordshire

College Lane, Hatfield, Herts.

AL10 9AB

United Kingdom

Tel: (0) 1707285122

Fax: (0) 1707285073

$\wedge$ corresponding author Email: K.J.Pine@herts.ac.uk

** The Open University, Milton Keynes, UK. 


\title{
A microgenetic analysis of the relationship between speech and gesture in children: Evidence for semantic and temporal asynchrony
}

\begin{abstract}
We present a microgenetic analysis of the gestures that children produce as they talk about a balance task. Children gesture spontaneously on this task and here their hand gestures are considered in relation to the accompanying speech. By close examination of 21 children's single sessions, and the 163 iconic gestures they produced (a mean of 7.6 gestures per child), it was found that gestures are rarely produced without speech. However, one third of the gestures the children produced conveyed different information to that expressed in their spoken explanations. Furthermore, children were found to convey information uniquely in gesture by expressing ideas in the manual modality that did not appear in their spoken explanations. Finally, in many cases children expressed an idea in gesture before they talked about it. These data suggest that gestures are integrally linked to the child's thinking and are an important and illuminating means of externalising cognition.
\end{abstract}

Keywords: Gesture - speech - microgenetic - iconic - mismatch - synchrony 


\section{Acknowledgement}

This research was supported by an Economic and Social Research Council grant, number R000223855, to Karen Pine and David Messer. We thank the children for their participation and the parents and teachers for their co-operation. 


\section{A microgenetic analysis of the relationship between speech and gesture in children: Evidence for semantic and temporal asynchrony}

A breakthrough in the study of children's gestures came with the discovery that it was relatively easy to interpret their meaning. Many gestures are iconic in nature (McNeill, 1992); that is, they are closely related to the semantic content of the accompanying speech. Thus, when talking about something going up the hands move upwards, when talking about something going round and round the hands move in a circular motion. Researchers studying children's understanding of the conservation of liquid task found that it was possible to code children's gestures according to which task variable they were conveying, e.g., the width of the container or the height of the liquid (Church \& Goldin-Meadow, 1986). Inter-rater reliability could also be established, with separate raters assigning the same meaning to gestures. The coding also has validity, in that the meanings that are attributed to the child on the basis of their gestures are the meanings the child had in mind (Garber, Alibali \& GoldinMeadow, 1998) Thus, within certain domains, the ideas that a child had could be gleaned not only from what they said, but also from how their hands moved. From this time on, researchers had another window into the mind of the child.

Through having this window, however, it was found that children's gestures did not always simply reflect the ideas expressed in their speech. By coding speech and gesture separately researchers noticed that the two were sometimes mismatched (Alibali \& Goldin-Meadow, 1993; Church \& Goldin-Meadow, 1986; McNeill, 1992; Perry et al., 1992). Alibali \& Goldin-Meadow (1993), for example, identified children 
who produced gesture-speech mismatches when asked to explain how they solved mathematical equivalence problems. These children sometimes expressed one method in speech but their gestures conveyed a different one. This and other studies provided evidence that a key characteristic of children's conceptual development involved cognitive variability, or the concurrent activation of two representations. Siegler (1996) states that cognitive variability is an essential component of development and that learning arises from a state where one has many different strategies in one's problem solving repertoire. This idea is substantiated by the research showing how children’s gesture speech mismatches predict learning. Children whose speech and gestures mismatched were more likely to benefit from instruction than children whose speech and gestures matched (Church, 1999; Church \& Goldin-Meadow, (1986), Perry et al., 1998, Pine, Lufkin \& Messer, 2004,). Therefore, these studies demonstrate the potency of gestures for telling us not just what the child is thinking about, but about their learning potential too. "Gesture may therefore be one of the best ways we have of discovering thoughts that are on the edge of a child's competence" (Goldin-Meadow, 2002, p.1399)

Gestures can convey the child's inner thoughts, provide additional information to that expressed in speech and signal to adults the child's knowledge state. It may also be that gesturing actually plays a role in the child's thinking processes. However, some theorists argue that gestures encode only what is encoded in speech, We consider two theoretical viewpoints concerning the functional role of gestures and the point in the speech production process where they are involved. 
The theoretical arguments that surround this question can be divided into two camps. There is one view that suggests that gesture is involved in generating the surface form of utterances (Butterworth \& Hadar, 1989; Krauss, 1998) and this argument is based on the Lexical Retrieval Hypothesis (see Alibali, Kita \& Young, 2000). According to this hypothesis, gestures facilitate the retrieval of lexical items from memory and thus play a direct role in the process of speaking. This hypothesis predicts that there would be a high degree of semantic synchronicity between the gestural and the spoken output, or the gesture's lexical affiliate. If gestures play an active role in lexical access then the content of the gesture would match the spoken expression and semantic synchrony should be high. This hypothesis would also predict temporal synchrony, or minimal asynchrony, as the surface form of the utterance would be contingent upon the gesture that activated it. "If gestures play a role in lexical retrieval they must stand in a particular temporal relationship to the speech they are presumed to facilitate" (Krauss, 1998, p.55). We would predict that, if a gesture helps the speaker retrieve a word, then the gesture would occur either prior to or simultaneous with, the production of its lexical affiliate. "It would be difficult to argue that a gesture helped a speaker retrieve a word if the gesture were initiated after the word had been articulated" (Krauss, 1998, p.55).

An alternative view of the functional role of gesture in speech production is the Information Packaging Hypothesis (Kita, 2000), which mitigates against the idea of gesture having a lexical affiliate. This view holds that gestures can convey ideas that are not compatible with the discrete and categorical format of spoken language; that they can convey ideas that do not fit into words. Ideas that are imagistic and analog may not lend themselves to verbal expression but nonetheless can 'leak' out in gesture 
(Alibali, Kita \& Young, 2000; Goldin-Meadow, 1999). According to McNeill \& Duncan (2000) gestures are 'global', they encompass the whole of the meaning, in contrast to speech, which is compositional and built up from arbitrary symbols.

A further role of gesture is to help organise different types of information, not at the level of speech production, but at the conceptual planning stage (Alibali et al., 2000). Gestures may also help in the representational redescription (RR) of spatial knowledge into a verbal format. In the RR model (Karmiloff-Smith, 1992) children are described as sometimes having representations that are not linguistically encoded and therefore not fully explicit. The children's gestures, therefore, may provide the key to discovering the nature of the unspoken ideas the child is working with and in the process of redescribing. "Expressing knowledge in gesture may therefore represent an important step in the redescription process that Karmiloff-Smith (1992) describes, a process culminating in explicit awareness" (Goldin-Meadow, 2001, p.28).

Most importantly, this view affords gestures a role not just in speech production but also in cognitive reorganisation, reasoning and thinking. "In brief, according to the Information Packaging Hypothesis, gesture plays a role in thinking." (Alibali et al. 2000, p.595). If gesture is involved in conceptual planning and can convey information that is incompatible with spoken language then the Information Packaging Hypothesis would allow for semantic asynchrony, i.e. different information being conveyed in speech than in gesture, or information being conveyed through one channel only. This view would also allow for a greater degree of temporal 
asynchrony, since gesture production would be less tied to speech production and need not occur contemporaneously.

We have considered the importance of gestures to children's thinking and to the construction and expression of their ideas, particularly during times of cognitive change. Many previous studies have sought to investigate these issues experimentally by employing a paradigm where gesture-speech mismatch is taken as a predictor of later learning gains. When learning gains are observed inferences are then drawn about the mechanisms responsible. However, it has been repeatedly shown in other studies of children's development that the adoption of a microgenetic approach will reveal more about underlying mechanisms of change. This method involves closely examining the very periods when children's knowledge is in transition. Since it is when children's knowledge is in transition that they are likely to gesture more, this method of close observation is also important for the study of gestures. Also, by applying this method we can take a closer look at the functional role of gestures in the process of speech production to determine whether they are involved at the speech generation or conceptual planning stage.

In this paper we adopt a microgenetic approach to study the gestures and speech of children as they are working with the balance beam task and during a time that their knowledge is in transition. This task has been employed extensively in previous work to test theories of cognitive development (Pine \& Messer, 1998, 1999, 2000, 2003; Pine et al., 1999, 2002). Here we subject the whole session to intense scrutiny rather than just measuring the beginning and end states. The children whose data are analysed were part of a larger study. All had incomplete knowledge of the balance 
task at the start and were in the process of acquiring the concept. This enabled us to capture their knowledge whilst it was in transition and study the speech-gesture relationship at this time of knowledge instability.

Critical to any analysis of the relationship between speech and gesture is the amount of temporal and semantic correspondence between them. Do they occur in perfect synchrony and convey the same information? An important question related to this concerns whether ideas emerge first in gesture or speech during on-line verbal and cognitive processing. This will speak to the issue of whether gesture is involved in the conceptual planning of an explanation or only in generating the surface form of the utterance. If gestures always convey the same information as speech, this would be consistent with the Lexical Retrieval Hypothesis. Yet studies from other domains have suggested that mismatches can occur or that information can be uniquely conveyed in gesture, which would accord more with the Information Packaging Hypothesis. We wish to explore this further within the micro domain of balance, a relatively unexamined area yet one that elicits discrete iconic gestures.

\section{Method}

\section{Design}

Videotaped sessions of children with the balance beam task were submitted to computer-based coding and analysis. In the sessions children attempted to balance six beams upon a fulcrum and were asked to explain why they thought the beams did or did not balance. The beams resembled wooden rulers and had weights at one or both ends. Hence some were symmetrical and balanced at their mid-point, those that were asymmetrically weighted balanced off-centre. The task for the child is to place the 
beam onto the fulcrum and adjust it until the balance point is reached. Children of this age frequently find the asymmetrical beams more difficult to balance but it is possible to observe their knowledge undergoing transition during a session. Of the children selected from a larger study to be analysed here, none achieved $100 \%$ behavioural success on the task, all failed to balance at least one of the beams. The sessions lasted between 4 and 8 minutes.

\section{Participants}

The participants were selected from a larger study. One class of 21 children was chosen at random. The mean age was 6.5 years, ranging from 6.0 to 7.75 years (sd $=$ $.34)$.

\section{Coding the sessions}

Our previous work identified consistent categories of discrete gestures produced by children talking about the balance beam task and has led to the establishing of a reliable coding system (Pine \& Lufkin, 2002; Pine, Lufkin \& Messer,2004). That scheme is utilised in the present study and, using the Observer system from Noldus, a computer-based programme that allows behaviours to be coded as they occur by means of keystrokes that correspond to a user-defined coding scheme. Several passes through the tapes were made, with the children's speech and gestures being coded separately before looking at any relationship between them. By scrutinising each child's session, moment-by-moment, the researchers coded all the children's speech and gestures independently using the codes summarised in Table 1. 


\section{Coding speech}

Speech was categorised by sentence, according to which aspect of the balance task the child was talking about, under the headings of 'weight', 'distance' or 'middle'. Any speech produced by the child that did not fit into one of these categories was coded as 'other'. This included talk unrelated to the task, or comments such as 'I don't know' (see Table 1)

\section{Coding gesture}

The children's iconic hand gestures were similarly coded. Our previous research identified the gestures that children produce to indicate the variables 'weight', 'distance' and 'middle' (Pine et al., 2004). Any other hand movements not falling into these categories were coded as 'other', this included both beats and self-adaptors. No distinction was made between beats and self-adaptors as our research question was principally concerned with the iconic, task related gestures the children produced. This type of coding differs from that used to scrutinise spontaneous speech, e.g. McNeill (1992), since the source data here are related to a very specific domain that is known to elicit task-related gestures in children. Whilst the gestural data are not as rich as with spontaneous speech produced, for example, when retelling a narrative, this does have the advantage of working with pre-coded variables that are known to appear in both speech and gesture when children talk about this domain,

(Table 1 about here) 


\section{Results}

After coding the content of the children's speech and gestures, time-lines were produced for each child's session with a breakdown of when each type of gesture and speech occurred. Inter rater reliability was established by having an independent researcher take a second pass through the data for 12 children's sessions. A Kappa value was obtained for each comparison. The Kappa values for the frequency of each type of gesture (and each type of speech) ranged from .72 - .96, with an average of 81\%. The Kappa values for the duration of each type of gesture and each type of speech ranged from .8 - .97, with an average of $89 \%$.

The 21 children produced a total of 163 gestures. All children produced at least one gesture and the most produced by a single child was 15, with a mean of 7.6 gestures per child (sd = 3.2).

Of the 163 gestures that were produced across all the sessions, 81 were coded as 'weight', 50 as 'middle', 9 as 'distance' and 23 as 'other'. Therefore $86 \%$ of the gestures could be accounted for by one of the iconic gestures in the coding scheme: only $14 \%$ were coded as 'other' (see Table 2). This provides a clear indication that the coding scheme accounted for most of the hand movements produced by children when explaining the balance beam task and that the majority of gestures produced were task relevant. This is in contrast to the children's utterances where almost half (47\%) were coded as 'other' i.e., during the sentence that accompanied a gesture they included no reference to any of the key task variables, weight, middle or distance. This suggests 
that children were more likely to indicate knowledge of the task variables in their gestures than in their speech.

(Table 2 about here)

Do gestures convey the same information as concurrrent speech?

When gestures were produced whilst the child was speaking, on 113 (69\%) of occasions they were coded as conveying the same information in gesture and speech during a single sentence. However, the remaining 50 (31\%) gestures did not match the accompanying speech. This means that, with reference to Table 1, on almost one-third of occasions, the child's speech was coded as belonging to one category (e.g. weight) but their gesture was coded as another category (e.g., distance).

Thus, although many of the gestures produced appeared to occur with speech, our closer analysis found speech and gesture did not always convey the same information. Furthermore, there were a few occasions when a referent appeared in gesture without any accompanying speech (see Figure 1 for an example: a block appears in the gesture line but none in the speech line).

Further examination was made of the 50 mismatches that the children produced (i.e., the $31 \%$ of occasions when the gesture did not match the accompanying sentence) to see whether the gestures conveyed information that was more advanced than the accompanying speech. Previous work (e.g. Pine \& Messer, 1999) has confirmed that children's developing knowledge follows a pattern of acquisition of the balance concept, with a hierarchy of increasingly sophisticated explanations from 'other' to 
middle, to weight and then distance. This enabled us to code the mismatching gestures as being either 'more advanced' or 'less advanced' than the accompanying speech. Forty of the 50 (80\%) mismatches involved gestures that were advanced than the accompanying speech. This differs significantly from what would be predicted by chance $\left(X^{2}=36 \mathrm{df}=1 \mathrm{p}=<.01\right)$. On many of these occasions the child's speech was coded as 'other' (i.e. lacking reference to any of the key variables) yet their gestures reflected knowledge about weight, distance, or middle.

What is the temporal relationship between speech and gesture?

Although it appeared that most of the time children gestured when they were speaking, this analysis considers whether gesture and speech have perfect temporal synchrony. Each child's session was captured by a timeline that summarised the time spent talking and the time spent gesturing. By examining the time-lines for speech (an example of a timeline is provided in Figure 1), and comparing them against those for gesture it was found that there was overlap between the speech and gesture lines on $88 \%$ of occasions. Children did not often gesture without speaking (there is one example of this in the time-line in Figure 1). The degree of temporal synchrony for matching speech and gesture is considered in the next section.

(Figure 1 about here)

Did gestures occur before, after or with the same speech?- analysis across each session

Although our analysis showed that, across the session, most gestures occurred with speech we decided to look more closely at the type gesture produced in relation to its lexical affiliate. Since not all children produced gestures for each variable, we focused 
on the first iconic gesture produced by each child, i.e. the first time in each child's session that a gesture was coded as 'weight', 'middle' or 'distance'. Then we looked within that session for when the same variable occurred in the child's speech. From this we were able to determine whether the gestured referent occurred before, after or with the corresponding spoken explanation, or even if it occurred in speech at all. For 12 children, their first iconic gesture overlapped with the time that the same variable was mentioned in speech, i.e. it was produced during the same sentence. For three children there was no such overlap, they conveyed a variable in gesture before speaking about it later in the session. A further six children conveyed information uniquely in gesture, i.e., it did not appear in the child's speech at all. Thus, 9 of the 21 children did not display close temporal synchrony of speech and gesture.

\section{Discussion}

The aim of this study was to microgenetically analyse sessions where children explained a balance task to illuminate the relationship between their speech and gestures. McNeill and Duncan (2000) claim that gestures and speech are systematically organised in relation to one another, that they will express the same underlying idea though not necessarily the same aspects of it. We found that, on as many as one third of occasions, children's speech and gestures conveyed different information and, on more than half of the instances we analysed, information was conveyed in gesture before speech.

Can one assume from this a model where the child's representation is translated into a mixture of speech and gesture? Our data are consistent with the Information 
Packaging Hypothesis, proposing that gestures can be generated independently of the linguistic formulation process. If gestures were contingent upon the speech production process, as the Lexical Retrieval Hypothesis states, it would be more difficult to account for the fact that often gestures convey different content and occur before speech. The Lexical Retrieval Hypothesis can account for the finding that gestures precede the corresponding utterance, since this account contends that gesturing facilitates retrieval of the word from lexical memory. However, the fact that gestures not only preceded utterances but also sometimes conveyed information that never appeared in the child's verbal utterance leads us to tentatively suggest either that lexical access failed (and the gesture was not facilitative) or, we think more plausibly, that the gesture was produced at the conceptual rather than the production stage. These gestures reflected the representation the child was working with, at a nonverbal level, but which was not yet found in speech.

The presence of both temporal and semantic asynchrony in our study militates against the Lexical Retrieval Hypothesis as the sole explanation for the role of gestures in speaking. These data suggest that, for the children on our study, gestures played a functional role in the conceptual planning of the idea, rather than in just generating the surface form of the utterance. This is not to deny that gestures can aid lexical retrieval in adults, as suggested by Frick-Horbury \& Guttentag (1998) but there is some evidence that this is not the case with pre-school children (Nicoladis, 2002). It is therefore possible that gestures have multiple functions and the relative importance of these changes with development (Goldin-Meadow, 2000). Our current work is examining this further by exploring how being prohibited from gesturing affects children's verbal dysfluency as well as their conceptual change (Pine et al., in prep). 
The children studied here all began the task with partial or incomplete knowledge of the balance task. By having some practice with the task and responding to probing questions from the experimenter they began the process of constructing for themselves ideas about how the task could be solved. This growth in understanding is analogous to the representational redescription process described by Karmiloff-Smith (1992). This model argues for an explicitation process underpinning and driving development and claims that there is a developmental gap between understanding and articulating knowledge. The gesture-speech dissociations found in this study concur with this model's claim that children frequently know more than they are able to articulate. Also, there was more precision in the children's gestures than in their speech overall. The majority (86\%) of the children's gestures were iconic, and related to the task variables, yet almost half $(47 \%)$ of the spoken utterances were vague and imprecise and could only be coded as 'other'. This is very clear evidence for gestures telling us more about the child's thoughts than speech. The finding that an idea can appear in gesture and not in speech, or can differ from speech, provides further support for the Information Processing Hypothesis. It is this semantic asynchrony, rather than temporal asynchrony, that is particularly problematic for the Lexical Retrieval Hypothesis.

We therefore argue that it can be informative and illuminating to use closely examine the gestures of children whilst they are in the process of learning, since their gestures are an integral part of the cognitive process. 'Gestures occur.... because they are part of the speaker's ongoing thought process. Without them thought would be altered or incomplete.' (McNeill, 1992). Research that focuses exclusively on children's 
articulated knowledge and ignores their gestures is in danger of missing a considerable part of what is going on in the child's mind. 


\section{References}

Alibali, M., \& Goldin-Meadow, S. (1993). Gesture-speech mismatch and mechanisms of learning: What the hands reveal about a child's state of mind. Cognitive Psychology,25, 468 - 523.

Alibali, M., Kita, S., \& Young, A. J. (2000). Gesture and the process of speech production: We think therefore we gesture. Language and Cognitive Processes, 15 , $593-613$.

Butterworth, G. \& Hadar, U. (1989), Gesture, speech, and computational stages: A reply to McNeill. Psychological Review, 96, 168 - 174.

Capirci, O., Iverson, J., Pizzuto, E. \& Volterra, V. (1996). Gestures and words during the transition to two-word speech. Journal of Child Language, 23, 3, 645-673.

Church, R. B. (1999). Using gesture and speech to capture transitions in learning. Cognitive Development, 14, 2, 313 -342.

Church, R. B., \& Goldin-Meadow, S. (1986). The mismatch between gesture and speech as an index of transitional knowledge. Cognition, 23, 43 - 71.

Frick-Horbury, D. \& Guttentag, R.E. (1998). The effects of restricting hand gesture production on lexical retrieval and free recall. American Journal of Psychology, 1, 43 $-62$. 
Garber, P., \& Goldin-Meadow, S. (2002). Gesture offers insight into problem-solving in adults and children. Cognitive Science, 26, 6, 817 - 831.

Garber, P. Alibali, M. \& Goldin-Meadow, S. (1998). Knowledge conveyed in gesture is not tied to the hands. Child Development, 65, 1, $75-84$.

Goldin-Meadow, S. (1999). The role of gesture in communication and thinking. Trends in Cognitive Science, 3, 11, 419 - 429.

Goldin-Meadow, S. (2001). Giving the mind a hand: The role of gesture in cognitive change. In J. L. McClelland \& R. S. Siegler (Eds) Mechanisms of cognitive development: Behavioural and neural perspectives. New Jersey: Lawrence Erlbaum Associates

Goldin-Meadow, S. (2002). Constructing communication by hand. Cognitive Development, 17, 3-4, 1385 - 1405.

Goldin-Meadow, S., Nusbaum, H., Kelly, S. D. \& Wagner, S. Explaining math: gesturing lightens the load. Psychological Sciences, 12, 516 - 522.

Karmiloff-Smith, A. (1992). Beyond Modularity: A Developmental Perspective on Cognitive Science. Cambridge, Mass: M.I.T. Press. 
Kita, S. (2000). How representational gestures help speaking. In D. McNeill (Ed.), Language and gesture (pp 162-185). Cambridge, UK: Cambridge University Press

Krauss, R. M. (1998). Why do we gesture when we speak? Current directions in psychological science, 7, 2, 54 - 60 .

McNeill, D. (1992)._Hand and mind: What gestures reveal about thought. Chicago: University of Chicago Press.

McNeill, D. \& Duncan, S. (2000). Growth points in thinking for speaking. In D. McNeill (Ed.), Language and gesture ${ }_{2}$ pp. 141 - 161. Cambridge: Cambridge University Press.

Nicoladis, E. (2002). Some gestures develop in conjunction with spoken language development and others don't: Evidence from bilingual preschoolers. Journal of Nonverbal behaviour, 26(4), 241 - 266.

Perry, M., Church, R. B., \& Goldin-Meadow, S. (1992). Is gesture-speech mismatch a general index of transitional knowledge? Cognitive Development, 7,_109 - 122.

Perry, M., Church, R. B., \& Goldin-Meadow, S. (1988). Transitional knowledge in the acquisition of concepts. Cognitive Development, 3, 359 - 400. 
Pine, K. J., \& Lufkin, N. (2002). Children’s discordant gestures as a predictor of learning gains: Evidence from a balance beam study. Poster presented at the Euresco Conference, Brain Development and Cognition, Naples, June, 2002.

Pine, K. J. \& Messer, D. J. (1998). Group collaboration effects and the explicitness of children’s knowledge. CognitiveDevelopment, 13 (1), 109 - 126.

Pine, K. J. \& Messer, D. J. (1999). What children do and what children know: Looking beyond success using Karmiloff-Smith’s RR framework. New Ideas in Psychology, 17, 17-30.

Pine, K. J. \& Messer, D. J. (2000). The effects of explaining another’s actions on children's implicit theories of balance. Cognition and Instruction, 18, 1, 37 - 54.

Pine, K. J. \& Messer, D. J. (2003). The development of representations as children learn about balancing. British Journal of Developmental Psychology, 21, 2, 285 - 301.

Pine, K. J., Messer, D. J. \& Godfrey, K. (1999). The teachability of children with naive theories: an exploration of the effects of two teaching methods. British Journal of Educational Psychology, 69 (2) $201-211$.

Pine, K. J., Messer, D. J., \& St. John, K. (2002). Children’s learning from contrast modelling. Cognitive Development, 17 (2) $1249-1263$.

Pine, K. J., Lufkin, N., \& Messer, D. J. (2004). More gestures than answers: Children learning about balance. Developmental Psychology, 40 (6) 1059-1067 
Pine, K. J., Kirk, E., Lufkin, N., \& Messer, D. J. (in prep). Linguistic and cognitive effects of gesture suppression in children.

Siegler, R. S., (1996). Emerging Minds. New York: OUP

Vygotsky, L. (1978). Mind in society: the development of higher psychological processes. Cambridge, MA: Harvard University Press. 
Figure 1: A timeline of one child's session.

The top line indicates each of the 6 beams the child was working with.

The second line indicates when the child was not talking (the darker areas) and talking (the lighter areas)

The third line indicates when the child was not gesturing (the lighter areas) and gesturing (the darker areas).

The thin vertical lines represent success (light) or failure (dark) at balancing the beam. At the bottom is the time, in minutes, from the beginning to the end of the session. 
Table 1: Codes for children's spoken and gestured explanations about the balance task.

\begin{tabular}{|c|c|c|}
\hline CODING & SPEECH & GESTURE \\
\hline OTHER & $\begin{array}{l}\text { The child talks about } \\
\text { something other than one of } \\
\text { the three variables (weight, } \\
\text { distance or middle) e.g., } \\
\text { colour or says 'I don't know' }\end{array}$ & $\begin{array}{l}\text { Non-iconic gestures, e.g., beats } \\
\text { (rhythmic movements keeping } \\
\text { time with speech) and self- } \\
\text { adapters (e.g. hair touching, head } \\
\text { scratching). }\end{array}$ \\
\hline MIDDLE & $\begin{array}{l}\text { The child speaks of the } \\
\text { importance of placing the } \\
\text { beam at its mid-point. }\end{array}$ & $\begin{array}{l}\text { The child points at the middle of } \\
\text { the beam with one or two hands. } \\
\text { In a two handed point the fingers } \\
\text { converge on the beam's centre. }\end{array}$ \\
\hline WEIGHT & $\begin{array}{l}\text { The child speaks about the } \\
\text { weight(s) on either side of } \\
\text { the beam or mentions } \\
\text { heaviness. }\end{array}$ & $\begin{array}{l}\text { The child closes all fingers } \\
\text { together so they are pointing } \\
\text { downwards. Gestures are } \\
\text { produced at one end of the beam, } \\
\text { typically the hand moves up and } \\
\text { down over one end of the beam. }\end{array}$ \\
\hline DISTANCE & $\begin{array}{l}\text { The child refers to the } \\
\text { distance of the weights from } \\
\text { the fulcrum or to the beam } \\
\text { being longer/shorter on one } \\
\text { side. }\end{array}$ & $\begin{array}{l}\text { The child moves one hand in a } \\
\text { sweeping movement from the } \\
\text { fulcrum to the end of the beam. } \\
\text { The hand is usually flat, palm } \\
\text { down and the movement is from } \\
\text { side to side. }\end{array}$ \\
\hline
\end{tabular}


Table 2: The frequency (and percentages) with which each type of code appeared in speech and gesture.

\begin{tabular}{|l|l|l|l|l|l|}
\hline CODE TYPE: & OTHER & MIDDLE & WEIGHT & DISTANCE & TOTAL \\
Coded in: & & & & & \\
\hline Speech & $102(47 \%)$ & $18(8 \%)$ & $87(40 \%)$ & $9(4 \%)$ & 216 \\
\hline Gesture & $23(14 \%)$ & $50(30 \%)$ & $81(49 \%)$ & $9(5 \%)$ & 163 \\
\hline & & & & & \\
\hline
\end{tabular}

